\title{
Pelvic Floor Muscles Contribution in Surgical Outcome of Children with High-type Anorectal Malformations
}

\author{
Stylianos Roupakias ${ }^{1}$, Xenophon Sinopidis $^{2}$ \\ ${ }^{1}$ Private Specialist Doctor, Volos, Greece; \\ ${ }^{2}$ Medical School, University of Patras, Patras, Greece \\ Received January 27, 2021; Accepted August 3, 2021.
}

Key words: Anorectal malformations - High-type - Muscle complex - Pelvic floor Outcome

\begin{abstract}
As a consequence of high-type anorectal malformations (ARMs) pathogenesis, the pelvic floor muscles remain severely underdeveloped or hypoplastic, the rectal pouch is located at the level or above the puborectalis sling, and the bowel terminates outside the sphincter muscle complex support. For children with high-type ARMs the ultimate objective of therapy is mainly to grow up having bowel continence function that is compatible with a good quality of life, and the final prognosis depends significantly on the grade of development of pelvic floor muscles and the successful entering of the anorectum fully within the support of the external anal sphincter due to intraoperative conservation of the puborectalis sling. Pelvic magnetic resonance imaging (MRI) has recently become the preferred imaging study for prediction of functional outcomes, since it can define the anatomy and evaluate the development of the sphincteric muscles before and after surgical correction. Based on recent literature and our clinical experience, we will discuss the relevance of pelvic floor muscles MRI to the clinical outcome of children with high type ARMs.
\end{abstract}

Mailing Address: Stylianos Roupakias, MD., 74 Hatziargiri str., Volos 38333, Greece; Phone: +306 973731 167; e-mail: stylroup@yahoo.gr 


\section{Introduction}

Anorectal malformations (ARMs) are among the most frequent congenital anomalies in paediatric surgery (Gangopadhyay and Pandey, 2015). They comprise a wide spectrum of anatomical presentations and associated anomalies involving the anorectal and urogenital system, sacral spine, and perineal musculature (Levitt and Peña, 2007; Bhatnagar, 2015a; Wood and Levitt, 2018). ARMs are divided into low, intermediate, and high types depending on whether the rectal pouch is located below, at the level, and above the pubococcygeal line (PC) and the ischiadic point (IP) respectively (Alamo et al., 2013). We used the older and rather obsolete classification of the three levels herein, to outline the topographic variations according to the level of the anomaly, implicating rather a more simplified and iconic approach for a radiological view of point, instead of the modern, widespread among paediatric surgeons, clinically-oriented and outcomeassociated classification of Pena (van der Steeg et al., 2015), thoroughly discussed at the meeting of Krickenbeck, Germany (Holschneider et al., 2005). There is a ventral displacement of the anal canal which opens either at the perineum or forms a fistula to the urogenital tract (Bhatnagar, 2015a), with the fistulous bowel terminating outside the support of the external anal sphincter (Kyrklund et al., 2017). At the same time, the pelvic floor muscles present varying degrees of hypoplasia ranging from normal musculature to absent muscle complex, depending on the severity of the lesion.

The pelvic floor is composed of a funnel-shaped sling of muscles with supporting and constricting functions, extending from the symphysis pubis to the coccyx, and from one lateral sidewall of the pelvic cavity to the other (Raizada and Mittal, 2008). The subcutaneous portion of the external anal sphincter (EAS) is located caudal to the internal anal sphincter (IAS), the superficial portion surrounding the distal part of the IAS, and the deep portion merging imperceptibly with the puborectalis muscle (Raizada and Mittal, 2008). In fact, EAS is not entirely a circular muscle, but is attached to the puboperineal muscle on either side (Ayoub, 1979). The puborectalis muscle is located between the superficial layers of the anal sphincters and the deep muscle layers of the levator ani muscle (Raizada and Mittal, 2008). Levator prostate or sphincter vaginae, ischiococcygeus, iliococcygeus and pubococcygeus, constitute the levator ani muscle, with some pubococcygeus fibers looped around the rectum forming the puborectalis as the most inferior part of the levator ani muscle group (Raizada and Mittal, 2008; Alamo et al., 2013). The term of a puborectal muscle sling isn't accepted by many pediatric surgeons performing this type of pediatric surgery. However, the future studies concerning this part of anorectal sphincter mechanism may confirm its existence.

Magnetic resonance imaging (MRI) has a leading role both before and after surgical correction of a high type ARM. Among others, MRI provides detailed structural information on the pelvic musculature anatomy and development of children with high type ARMs. Ultimate objective of management is mainly the 
outcome of a bowel within the best achievable results in anatomy and continence (Alamo et al., 2013), as high-type anomalies are characterized by major structural deficits, non-compatible with a fair quality of life. This review aims to outline essential points regarding the functional anatomy of the pelvic muscles in high-type ARMs.

\section{Evolution of surgical treatment in relation to the pelvic floor muscles}

Stephens in 1953, the first paediatric surgeon who studied the anatomy of the pelvis in children with ARMs, concluded that the puborectalis sling is the key part of the sphincter mechanism to achieve postoperative bowel control (Zaiem and Zaiem, 2017). The pull-through techniques were considered as blind techniques as they did not ensure accurate placement of the rectum at the center of the muscle complex. De Vries and Pena in 1982 introduced the posterior sagittal anorectoplasty (PSARP) technique, which ensured the placement of the rectum inside the sphincter mechanism (Zaiem and Zaiem, 2017). It was learned from the procedure that there is a strong funnel-like muscle structure that forms the sphincter mechanism, with its upper portion of horizontal muscle fibers referred as the levator muscle, and its lower portion (named by Pena as the muscle complex) consisted mainly of vertical fibers running parallel to the rectum (Zaiem and Zaiem, 2017). PSARP also underlined the role of the muscle complex as a functional prominent structure (Zaiem and Zaiem, 2017; Patel et al., 2018). Therefore, adequate placement of the neorectum not only through the puborectalis sling but also within the EAS is necessary for an adequate functional outcome (Bhuynan et al., 2015). In an attempt to give the muscle complex its due respect by keeping it intact and restored around the new pulled rectum, sphincter saving anorectoplasty (SSARP) and muscle complex saving posterior sagittal anorectoplasty (MCSPSARP), have been described (Bhuynan et al., 2015).

The later introduced laparoscopically assisted anorectal pull-through (LAARP) technique for the high-type ARMs, aiming to reduce the amount of posterior dissection of the sphincter mechanism required for the accurate placement of the neorectum into the muscle complex under laparoscopic vision (Georgeson et al., 2000). The avoidance of division of the vertical muscle complex owed to the longitudinal cutting of the sphincter muscle complex with the PSARP technique, resulted in the minor disturbance of the muscle innervations (Bhuynan et al., 2015). The laparoscopic approach resulted in better sphincter symmetry, and lesser irregularity and perirectal fibrosis compared to PSARP (Bhatnagar, 2015c). A limitation of the method is that the narrow path of the vertical muscle fibers between the pelvic floor and the perineal parasagittal muscle fibers cannot be visualized (Raschbaum et al., 2010). This raises the likelihood of deviation from the course of the central portion of the vertical muscle complex while performing pull-through of the rectal segment to the perineum (Raschbaum et al., 2010). 


\section{Preoperative pelvic floor muscle MRI}

MRI has a defined role in the imaging protocol of ARMs (Madhusmita et al., 2018), because of its lack of ionizing radiation, excellent intrinsic contrast resolution, and multiplanar imaging capabilities (Bhatnagar, 2015c). Disadvantages comprise an occasional lack of expertise or access to the technique, a relative high cost, and the need for sedation (in neonates and young infants the examination must be done with general anaesthesia or by the way of a "feed and wrap" i.e., sleeping after eating) (Podberesky et al., 2013). Pelvic MRI has recently become the preferred imaging study for defining the anatomy and evaluating the size, the morphology, and the grade of development of the sphincteric muscles before surgical correction (Weinstein et al., 2009; Alamo et al., 2013; Bhatnagar, 2015b). Functional prognosis of high type ARM can be predicted, since children with an underdeveloped sphincter muscle complex are likely to be incontinent (Madhusmita et al., 2018).

In neonates and infants with ARMs a pelvic MRI protocol should include T1 and T2 weighted images in coronal, sagittal, and axial (transverse) planes. The anatomic characteristics of the sphincter muscle complex are clearly demonstrated by routine T1 and T2 weighted images but are depicted more affluently on T2 (Tang et al., 2006). Restrained fat signal can be applied for better muscle visualization (Tang et al., 2006). No anatomic distinction between the individual muscles of the levator ani or the EAS can be routinely detected by MRI, nevertheless their distinction is of no clinical value in the setting of ARM correction (Podberesky et al., 2013). The pubococcygeal plane corresponds to the attachment level of the levator ani muscle to the pelvic wall, extends from the upper border of the os pubis to the os coccyx, and includes the prostate in males or the cervix in females and the rectum (Alamo et al., 2013). In the axial images of this plane, the puborectalis muscle appears as a triangular band surrounding the rectum posterolaterally (Alamo et al., 2013; Madhusmita et al., 2018). A well-developed levator ani is seen clearly on coronal images as a sling-like structure supporting the rectal ampulla (Madhusmita et al., 2018). The levator ani muscle fibers are intraoperatively recognized as vertical fibers attached to the sacrum and to the rectal wall. The ischial plane following the line joining the lowest points of the ischial tuberosities represents the deepest point of the funnel of the levator ani muscles (Alamo et al., 2013). The oval shaped external anal sphincter posteriorly can be clearly observed in this plane and the deep portion of the EAS can be distinguished in axial images from the cranially located directly adjacent puborectalis muscle, appearing to overlap the puborectalis muscle bundles (Alamo et al., 2013). The EAS is seen as a posterior curved band-like structure in sagittal images, with fibers extending in the parasagittal images, and as an oval structure symmetrically surrounding the anal canal in the axial images (Madhusmita et al., 2018). In the midsagittal plane, the EAS encircles the anal canal both anteriorly and posteriorly, with the lower anterior part extending ventrally and the posterior extending to and connecting with the coccyx, caudally to the puborectalis muscle (Alamo et al., 2013). 
MRI images are evaluated for the subjective developmental state of the sphincter muscle complex, in particular the length, width, and thickness of the puborectalis and EAS muscles (Tang et al., 2006; Madhusmita et al., 2018). Although some studies used objective measurements of the sphincteric and levator sling muscles to describe their overall quality on MRI, a subjective good, moderate, or poor assessment is typically adequate (Podberesky et al., 2013; Madhusmita et al., 2018). Deviation from the defined as normal appearance is evaluated as fair or moderate. When the muscle fibers are poorly visualized, they quality is graded as poor (Madhusmita et al., 2018). The higher the ARM, the poorer the sphincter muscle complex development is seen in MRI (Kyrklund et al., 2017; Madhusmita et al., 2018). In children with low ARMs, the well-developed sphincteric muscles usually demonstrate normal or almost-normal size and morphology at MRI (Alamo et al., 2013). Moreover, the rectum is usually located within most of the sphincters, except an anteriorly mislocated lower part in some cases (Alamo et al., 2013). In children with high type ARM, the underdeveloped sphincteric muscles are frequently asymmetric and highly hypoplastic (Alamo et al., 2013). Most females with a cloacal anomaly have hypoplastic and underdeveloped levator ani muscle and EAS, and these with a longer common canal present highly to extremely hypoplastic and underdeveloped levator ani muscle, and almost unrecognizable EAS (Alamo et al., 2013).

There are MRI measurement indexes that can be taken as quantitative criterion for poor developmental state of pelvic floor muscles (Madhusmita et al., 2018). These indexes could be used as clinical predictive factors for surgical outcome of children with high type anorectal malformations. The relative width of the puborectalis muscle (RWPR) and EAS (RWEAS) on a transverse plane, are defined as the ratio of the total width of muscle (as the sum of both left and right muscle width of the rectum or anal canal) and the half distance of ischial tuberosities (Madhusmita et al., 2018). The relative length of puborectalis muscle (RLPR) and EAS (RLEAS) on a sagittal plane, are defined as the ratio of the length of muscle and the length of the pubococcygeal line (Madhusmita et al., 2018). When PRWR is $<0.18$ and EASWR $<0.15,71 \%$ of the patients with ARMs suffer from anal incontinence postoperatively (Madhusmita et al., 2018). When PRWR is $>0.18$ and EASWR $>0.15,91 \%$ of the patients with ARM have good continence, and the poor continence in the remaining patients is mainly due to constipation (Madhusmita et al., 2018). All paediatric surgeons who perform ARMs corrective surgery are familiar with the widespread index known as sacral ratio, based on the sacral length with anatomic pelvic bone landmarks, and measured with lateral pelvic radiographs. Values below 0.4 have a predictive value of poor functional outcome. Krois et al. (2021) in a retrospective cohort study, demonstrated recently that the sacral ratio can be calculated on MRI with a good reliability, also providing the advantage of less exposure to ionizing radiation. 


\section{Postoperative pelvic floor muscle MRI}

Except the musculature developmental status, appropriate placement of the rectal pull-through within the levator muscle, which is the most important factor of continence, and the EAS, is critical for optimal postoperative bowel continence (Bhatnagar, 2015b; Bhuynan et al., 2015). MRI is the optimal method for the evaluation of postoperative fecal incontinence, the study of complications, and the design of management in cases of consideration of potential reoperation (Podberesky et al., 2013; Bhatnagar, 2015c; Madhusmita et al., 2018). MRI evaluation includes the developmental quality and the postoperative shape of the striated muscle complex, the positioning of the neorectum in relation to the muscles, the anorectal angle, the peritoneal fat herniation, and the postoperative muscle scarring, outlining as a main task to show a mislocated rectum and the damaged sphincteric muscles (Podberesky et al., 2013; Yong et al., 2013; Raman et al., 2015).

T1 and fast or turbo spin-echo T2 weighted sequences are applied in the axial/ sagittal/coronal planes without fat saturation, with the surgeon interested and focused in the midsagittal sections because it is the plane used for the operative approach (Eltomey et al., 2008). Anterior misplacement of the neorectum in the EAS, and lateral misplacement of the neorectum in the puborectalis muscle, are the most common surgical errors observed (Madhusmita et al., 2018). The sphincter muscle complex is best seen in the axial images at the level of the symphysis pubis and below (Eltomey et al., 2008; Raman et al., 2015). Axial and coronal images show better a side to side displacement of the bowel, while sagittal images help in the assessment of an anteroposterior displacement of the bowel in relation to the sphincter (Eltomey et al., 2008; Raman et al., 2015).

The measurement of the thickness/continuity/regularity of the pelvic muscles is actually a subjective assessment, based on the internal symmetry from side to side and the comparison with normal pelvic musculature in similar aged, healthy patients (Podberesky et al., 2013; Farghaly et al., 2018). Development of the striated muscles is defined as good if the muscle has a regular shape and normal thickness, intermediate dysgenesis if the muscle is intact with reduced thickness, and poor dysgenesis if the muscle is disrupted or deformed (Yong et al., 2013). In some cases, the bowel is properly positioned but mesenteric fat that is inadvertently pulled with bowel through the sphincter during the initial repair interferes with the continence mechanism (Eltomey et al., 2008). Peritoneal fat herniation occurs in cases of colonic pull-through, if a rectum must be excised if too short or ischemic, and replaced by colon. Fat herniation weakens the rectal fullness sensation and voluntary muscles stretching ability, by damaging the integrity of the muscle complex (Yong et al., 2013).

Extensive postoperative muscle scarring may cause defecatory dysfunction, and due to its stiff appearance and lower T2 signal can be easily differentiated from normal muscle tissue (Yong et al., 2013). 
Children with a lower obtuse anorectal angle have a better clinical outcome (Desai et al., 2018). The normal angle is $<90^{\circ}$ and any angle $>100^{\circ}$ is considered abnormal (Farghaly et al., 2018). The changes in the anorectal angle are predominantly related to the development status of puborectalis muscle (Raizada and Mittal, 2008; Wahab et al., 2017).

Descending perineum syndrome may be caused by damage to the muscles and ligaments of the pelvic floor during the operation (Levin, 2018), in combination with coexisting hypoplastic muscle weakness. The descent of the pelvic floor is mostly related to the pubo/ileo/ischio-coccygeous muscles (Raizada and Mittal, 2008). A rectal descent (descent of the anorectal junction below the pubococcygeal line) is classified as mild $(2-4 \mathrm{~cm})$, moderate $(4-6 \mathrm{~cm})$, or severe $(>6 \mathrm{~cm})$ (Yong et al., 2013).

Children with abnormally located neorectum and/or increased anorectal angle and/or peritoneal fat herniation and/or pelvic floor dysfunction and/or extensive scarring often require further surgery (Yong et al., 2013). In children with isolated maldevelopment of striated muscle complex, conservative treatment should be offered to relieve symptoms (Yong et al., 2013).

\section{What about the internal anal sphincter?}

The practical significance of the intrinsic circular muscle located at the rectal end, known as internal anal sphincter (IAS) has remained controversial in high type anomalies in the relevant literature (Mirshemirani et al., 2009). A functional IAS in anorectal manometry, as indicated by a positive rectoanal inhibitory reflex, has been associated with improved functional outcomes in children who have high or intermediate ARMs by certain researchers (Husberg et al., 1997; Kyrklund et al., 2017). Animal models of ARMs have shown dysplastic IAS with variability in shape and size (Cleeve et al., 2011). Studies reported that the intrinsic muscle layers or the muscle in total are characteristically abnormal and hypoplastic in neonates with high type ARMs (Meier-Ruge and Holschneider, 2000). Functional studies in children with different levels of ARM demonstrated an intact postoperative recto-anal inhibitory reflex (Cleeve et al., 2011). A smooth muscle layer can also be seen close to the opening of the fistula from the rectum to the urogenital tract (Husberg et al., 1997). In addition, electrical field stimulation on smooth muscle strips from the caudal part of the rectal pouch have shown the same characteristics as that of IAS (Husberg et al., 1997; Desai et al., 2018). It was speculated it maybe of functional significance (Cleeve et al., 2011). If the fistula was preserved and transposed to the normal position of the anal canal, an IAS function was anticipated in most cases (Mirshemirani et al., 2009). These findings favoured the conservation of the distal part of the fistula in severe ARMs (Kyrklund et al., 2017). It was stated that the most caudal rectal internal circular muscle fibers have the potential to degenerate and develop as IAS after been transplanted, even though they are histologically loose and hypoplastic at the definitive surgery and contribute to the improvement of passive 
continence in the late postoperative period (Mirshemirani et al., 2009). We believe that IAS does not play an important role, particularly in the so-called high ARMs. Occasionally, its resection together with a fistula and the adjacent portion of the rectum if aganglionic is mandatory.

MRI allowed the possibility to show the anatomic evidences of an IAS-like structure encircling the anal canal in all patients operated with PSARP for high or intermediate ARMs, independently of the severity of the malformation or the postoperative physiological IAS function (Husberg et al., 1997). These fibers are notably more irregular, with variations in thickness in different directions or levels in the anal canal and present a larger surface area than those of normal children (Husberg et al., 1997).

\section{What is new with pelvic floor muscles MRI in the anorectal malformations?}

MRI guided LAARP is now available. An MRI compatible needle is used to penetrate the perineal skin at the central site of the parasagittal muscle contraction as determined by direct muscle stimulation (Raschbaum et al., 2010). Serial scans are obtained in axial, coronal, and sagittal planes as the needle is advanced cephalad to remain within the central portion of the vertical muscle complex until the peritoneal floor is penetrated (Raschbaum et al., 2010). This method promises better outcome, because of accurate placement of the rectal pull-through within the muscles and less muscle surgical trauma.

In recent years dynamic MRI has been used in older cooperative children and adolescents with pelvic floor dysfunction and fecal incontinence (Boemers et al., 2006; Yong et al., 2013; Wahab et al., 2017; Levin, 2018). Minimal function (movement) of pelvic floor during evacuation attempt, and poor elevation or asymmetrical movement of the levator ani during squeeze can be identified postoperatively (Boemers et al., 2006).

\section{Conclusion}

Pelvic floor muscle MRI is considered as required for high-type ARM assessment. The introduction of preoperative and postoperative $M R I$ in the evaluation and management of children with high type ARMs resulted in more detailed anatomical knowledge and better understanding of the clinical importance of the striated muscle complex of the pelvic floor. We may at last identify details of the preoperative congenital deviations and the postoperative outcomes under a powerful light instead of speculating in the shadows. Operative guidance is a significant novelty in the use of pelvic floor muscle MRI for the surgical correction of ARMs.

\section{References}

Alamo, L., Meyrat, B., Meuwly, J. Y., Meuli, R. A., Gudinchet, F. (2013) Anorectal malformations: Finding the pathway out of the labyrinth. Radiographics 33, 491-512.

Ayoub, S. F. (1979) Anatomy of the external anal sphincter in man. Acta Anat. (Basel) 105, 25-36. 
Bhatnagar, S. (2015a) Anorectal malformations (Part 1). J. Neonatal Surg. 4, 7.

Bhatnagar, S. (2015b) Anorectal malformations (Part 2). J. Neonatal Surg. 4, 25.

Bhatnagar, S. (2015c) Anorectal malformations (Part 3). J. Neonatal Surg. 4, 29.

Bhuynan, D., Agarwal, S., Khongsi, P., Padmanabhan, A. (2015) Magnetic resonance evaluation of anorectal malformation cases treated by posterior sagittal anorectoplasty. J. Evol. Med. Dent. Sci. 4, 3995-4007.

Boemers, M. T., Ludwikowski, B., Forstner, R., Schimke, C., Ardelean, A. M. (2006) Dynamic magnetic resonance imaging of the pelvic floor in children and adolescents with vesical and anorectal malformations. J. Pediatr. Surg. 41, 1267-1271.

Cleeve, S., Lawson, J., Martin, J., Ward, H. (2011) Immunohistochemical confirmation of the presence of smooth muscle in the normal neonatal anorectum and in neonates with anorectal malformations. Pediatr. Surg. Int. 27, 1069-1074.

Desai, S., Choudhury, H., Joshi, P., Pargewar, S. (2018) Magnetic resonance imaging evaluation after anorectal pull-through surgery for anorectal malformations: a comprehensive review. Pol. J. Radiol. 83, e348-e352.

Eltomey, M., Donnelly, L., Emery, K., Levitt, M., Pena, A. (2008) Postoperative pelvic MRI of anorectal malformation. AJR Am. J. Roentgenol. 191, 1469-1476.

Farghaly, T. Y., Ettaby, N. A., Waheeb, M. S., Moghazy, M. K., El Tomey, A. M. (2018) Postoperative MRI for anorectal malformations. Egypt. J. Radiol. Nucl. Med. 49, 959-970.

Gangopadhyay, A. N., Pandey, V. (2015) Anorectal malformations. J. Indian Assoc. Pediatr. Surg. 20, 10-15.

Georgeson, K. E., Inge, T. H., Albanese, C. T. (2000) Laparoscopically assisted anorectal pull-through for high imperforate anus - A new technique. J. Pediatr. Surg. 35, 927-931.

Holschneider, A., Hutson, J., Pena, A., Beket, E., Chatterjee, S., Coran, A., Davies, M., Georgeson, K., Grosfeld, J., Gupta, D., Iwai, N., Kluth, D., Martucciello, G., Moore, S., Rintala, R., Smith, E. D., Sripathi, D. V., Stephens, D., Sen, S., Ure, B., Grasshoff, S., Boemers, T., Murphy, F., Söylet, Y., Dübbers, M., Kunst, M. (2005) Preliminary report on the International Conference for the Development of Standards for the Treatment of Anorectal Malformations. J. Pediatr. Surg. 40, 1521-1526.

Husberg, B., Rosenborg, M., Frenckner, B. (1997) Magnetic resonance imaging of anal sphincters after reconstruction of high or intermediate anorectal anomalies with posterior sagittal anorectoplasty and fistula-preserving technique. J. Pediatr. Surg. 32, 1436-1442.

Krois, W., Palmisani, F., Gröpel, P., Feil, P., Metzelder, M. L., Patsch, J. M., Reck-Burneo, C. A. (2021) Assessment of sacral ratio in patients with anorectal malformations - Can magnetic resonance imaging replace conventional radiographs? - Sacral-ratio from MRI. J. Pediatr. Surg. S0022-3468(21)00033-6 (Epub ahead of print)

Kyrklund, K., Pakarinen, M. P., Rintala, R. J. (2017) Long-term bowel function, quality of life and sexual function in patients with anorectal malformations treated during the PSARP era. Semin. Pediatr. Surg. 26, 336-342.

Levin, D. M. (2018) Pathophysiology and diagnosis of descending perineum syndrome in children. Pelviperineology 37, 52-56.

Levitt, M. A., Peña, A. (2007) Anorectal malformations. Orphanet J. Rare Dis. 2, 33.

Madhusmita, Ghasi, R. G., Mittal, M. K., Bagga, D. (2018) Anorectal malformations: Role of MRI in preoperative evaluation. Indian J. Radiol. Imaging 28, 187-194.

Meier-Ruge, W. A., Holschneider, A. M. (2000) Histopathologic observations of anorectal abnormalities in anal atresia. Pediatr. Surg. Int. 16, 2-7.

Mirshemirani, R. A., Sadeghian, S., Zamir, R. F. (2009) Internal anal sphincter, and late clinical outcome in patients with high type anorectal malformation. Act. Med. Iran. 47, 139-142.

Patel, J. L., Memon, M. A., Sharma, S., Verma, J. (2018) Assessment of post-surgical functional outcome in children with anorectal malformation. Int. Surg. J. 5, 1286-1290. 
Podberesky, J. D., Towbin, J. A., Eltomey, A. M., Levitt, A. M. (2013) Magnetic resonance imaging of anorectal malformations. Magn. Reson. Imaging Clin. N. Am. 21, 791-812.

Raizada, V., Mittal, R. K. (2008) Pelvic floor anatomy and applied physiology. Gastroenterol. Clin. North Am. 37, 493-509.

Raman, S. V., Agarwala, S., Bhatnagar, V., Gupta, K. A. (2015) Correlation between functional outcomes and postoperative pelvic magnetic resonance imaging in children with anorectal malformation. J. Indian Assoc. Pediatr. Surg. 20, 116-120.

Raschbaum, R. G., Bleacher, C. J., Grattan-Smith, J. D., Jones, A. R. (2010) Magnetic resonance imaging-guided laparoscopic-assisted anorectoplasty for imperforate anus. J. Pediatr. Surg. 45, 220-223.

Tang, S. T., Wang, Y., Mao, Y. Z., Tong, Q. S., Li, S. W., Cao, Z. Q., Ruan, Q. L. (2006) MRI of anorectal malformations and relationship of the developmental state of the sphincter muscle complex with fecal continence. World J. Pediatr. 3, 223-230.

van der Steeg, H. J. J., Schmiedeke, E., Bagolan, P., Broens, P., Demirogullari, B., Garcia-Vazquez, A., Grasshoff-Derr, S., Lacher, M., Leva, E., Makedonsky, I., Sloots, C. E., Schwarzer, N., Aminoff, D., Schipper, M., Jenetzky, E., van Rooij, I. A., Giuliani, S., Crétolle, C., Holland Cunz, S., Midrio, P., de Blaauw, I. (2015) European consensus meeting of ARM-Net members concerning diagnosis and early management of newborns with anorectal malformations. Tech. Coloproctol. 12, 181-185.

Wahab, S., Balkrishna, Z. T., Khan, A. R., Ahmad, I. (2017) MRI following PSARP in children with anorectal malformations: Differences that matter! Int. J. Cont. Med. Surg. Radiol. 2, 154-157.

Weinstein, M. M., Pretorius, D. H., Jung, S.-A., Nager, C., Mittal, R. K. (2009) Transcutaneous 3-dimensional ultrasound imaging: A novel technique to detect anatomical defects in the muscles of anal sphincter complex. Clin. Gastroenterol. Hepatol. 7, 205-211.

Wood, R. J., Levitt, M. A. (2018) Anorectal malformations. Clin. Colon Rectal Surg. 31, 61-70.

Yong, C., Ruo-yi, W., Yuan, Z., Shu-hui, Z., Guang-rui, S. (2013) MRI findings in patients with defecatory dysfunction after surgical correction of anorectal malformation. Pediatr. Radiol. 43, 964-970.

Zaiem, M., Zaiem, F. (2017) Muscle complex saving posterior sagittal anorectoplasty. J. Pediatr. Surg. 52, 889-892. 